Abstract P2 Table 1 The clinical and laboratory manifestations for the entire period of the disease

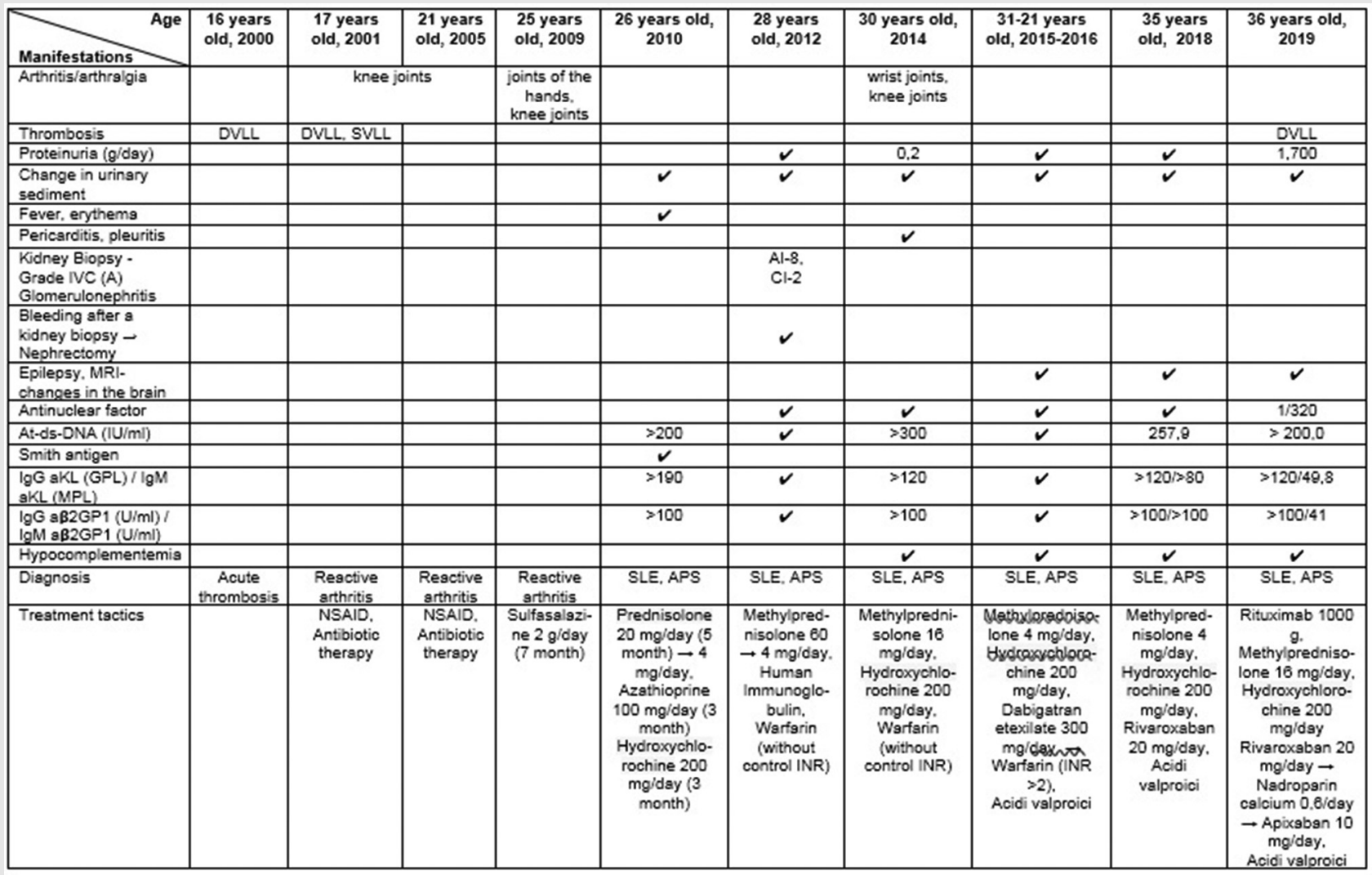

-OVLL - deep veins lower leg. SVLL - superficigl veins lower leg. Al - gctivity index, CI - chronicity index, SLE - systemic lupus erythematosus, APS - antiphospholipid syndrome, NSAID - nonsteroidgl anti-inflammstory drugs, INR - internstionsl norms lized ratio

clinical finding are: active lupus nephritis of a kidney, relapse of venous thrombosis as a result of APS, thrombotic microangioencephalopathy, cognitive impairment and somatogenic asthenodepressive syndrome.

Conclusions This clinical case demonstrates a patient with SLE presented in clinical features thrombosis, lupus glomerulonephritis class IV, AI 8, CI 2, high immunological disorders and concomitant APS. The difficulty in treating the patient is the consistently high immunological activity of the disease, the resistance of the therapy cytostatic and glucocorticoids, the inefficiency and intolerance of a number of oral anticoagulants: dabigatran etexilate and rivaroxaban - relapses of thrombosis, warfarin - the inability to reach the target levels of the international normalized ratio (INR) due to recurrent nosebleeds.

\section{P3 IS THERE A RELATIONSHIP BETWEEN LUPUS ANTICOAGULANT AUTOANTIBODY AND FATIGUE IN JSLE?}

${ }^{1}$ Sara Ganhão, ${ }^{2}$ Beatriz Silva, ${ }^{1}$ Mariana Rodrigues, ${ }^{1}$ Francisca Aguiar, 2,3 Margarida Figueiredo-Braga, ${ }^{1,2}$ Iva Brito. ${ }^{1}$ Young Adult and Pediatric Rheumatology Unit, Centro Hospitalar e Universitário do Hospital de São João, Porto; ${ }^{2}$ Faculty of Medicine, University of Porto, Porto; ${ }^{3}$ Dept. of Clinical Neurosciences and Mental Health, Porto, Portugal

10.1136/lupus-2020-eurolupus.52

Background Fatigue is a common symptom in systemic lupus erythematosus (SLE) and is associated with poorer quality of life and higher depression scores. Lupus anticoagulant (AcL) refers to a subset of autoantibodies to phospholipids interfering with clotting tests, which major clinical association is thrombosis. Physical activity reduces thrombotic risk in general and is a potential therapeutic strategy for managing fatigue in SLE patients. Purpose: To compare fatigue outcomes according to AcL status in juvenile-onset SLE (jSLE).

Methods A cross-sectional sample of jSLE patients, currently aged $\geq 16$ years, completed SF-36, HADS and FSS questionnaires between October 2018- May 2019. The study was approved by the local Ethics Committee. All patients fulfilled both 2012 and 2019 EULAR/ACR classification criteria for SLE. Juvenile-onset was defined as age at diagnosis $<18$ years. Demographics and clinical characteristics were collected. Statistical analysis was performed with SPSS ${ }^{\circledR}$.

Results jSLE patients $(n=30)$ were studied (90\%female) with median age of 21 years, range 16-35, with mean (SD) age of diagnosis of $15.8 \pm 2.1$. Mean values (SD) of psychological assessment were: FSS $4.27 \pm 1.36$; Physical health SF36 of $66.8(9.9)$ and Mental health SF-36 of 68.9 (17.5). $56.7 \%$ jSLE showed clinically relevant fatigue, $63.3 \%$ anxiety and $13.3 \%$ depression. Four patients (13.3\%) had positive AcL autoantibody. Those had lower Fatigue Severity Scale (FSS) average scores: $3.0(0.16)$ vs $4.5(0.28)$, comparing to those who were negative for AcL $(p=0.036)$. Likewise, positive AcL jSLE patients had better results in SF-36 Physical Health questionnaires $(p=0.034)$.

Conclusions The relationship between AcL status and fatigue was not previously known. We hypothesize that the present finding may be related to physical activity, a major indication 
for reducing thrombotic risk and fatigue in jSLE patients. Further studies are needed to address this issue.

\section{P4 ANTIPHOSPHOLIPID SYNDROME IN PATIENTS WITH ACUTE MYOCARDIAL INFARCTION}

${ }^{1}$ Mikel Jordhani, ${ }^{2}$ Dorina Ruci, ${ }^{3}$ Joana Seiti. ${ }^{1}$ Dept. Internal Medicine, Rheumatology Clinic, UHC Mother Teresa, Tirana; ${ }^{2}$ Dept. Internal Medicine, Rheumatology Clinic, UHC Mother Teresa, Tirana; ${ }^{3}$ Dept. Cardiovascular Diseases, Cardiovascular Reanimation Clinic, UHC Mother Teresa, Tirana, Albania

\subsection{6/lupus-2020-eurolupus.53}

Background Antiphospholipid Syndrome (APS) is a known cause of thrombotic disorders, including Acute Myocardial Infarction (AMI). Although its incidence in AMI patients is not known, it may be an important factor in precipitating infarction, especially in younger adults.

Methods This is a case-control study consisting in 73 patients with Acute Myocardial Infarction, hospitalized at Cardiovascular Reanimation Clinic from 10 December 2015- 10 October 2019. All patients included in the study were from 23 to 50 years old. In the case-group were included 24 patients with Antiphospholipid Syndrome and Acute Myocardial Infarction, and 49 patients were included in the control group, which consisted only in patients with AMI, with no presence of APS. In every patient were gathered data such as complete blood count + ESR, Antinuclear Antibodies (ANA), Anti-cardiolipin antibodies (ACA), C3 and C4 complement fraction, Anti-ds-DNA, CRP and Ejection Fraction (EF) in echocardiography. Female patients were asked about their abortion history. As recommended in the guidelines, in positive results for APS, ACA levels were repeated after 12 weeks in order to establish the diagnosis. All data were gathered and statistically analyzed using Excel 2010 and IBM SPSS.

Results After comparing all gathered data, it was found that the patients with APS and AMI had a more significant tendency to have C3 hypocomplementemia $(p=0.006)$, thrombocytopenia $(\mathrm{p}=0.002)$, a lower ejection fraction on transthoracic echocardiography $(p=0.04)$ and a more elevated number of abortions before acute myocardial infarction $(p=0.03)$ in comparison to the controls.

Conclusions From our study it was found that APS is not rare in young adults with AMI. It should be always suspected in young patients with no cardiovascular risk factors and there may be a characteristic clinical and laboratory picture in patients with AMI, which may suggest the APS diagnosis.

Acknowledgements The authors declare no conflict of interest.

\section{P5 RECOGNIZING THE DISEASE, TREATING THE PATIENT: A MIXED-METHOD EVALUATION OF CARE FOR THE ANTIPHOSPHOLIPID SYNDROME (APS) IN THE NETHERLANDS}

\footnotetext{
${ }^{1}$ Mirthe J Klein Haneveld, ${ }^{1}$ Caro Lemmen, ${ }^{1}$ Tammo Brunekreef, ${ }^{2}$ Marc Bijl, ${ }^{3}$ Gerard Jansen, ${ }^{4}$ Karina de Leeuw, ${ }^{1}$ Julia Spierings, ${ }^{1}$ Maarten Limper, on behalf of the ARCH study group. ${ }^{1}$ Dept. of Rheumatology and Clinical Immunology, University Medical Centre Utrecht, Utrecht; ${ }^{2}$ Dept. of Internal Medicine and Rheumatology, Martini Hospital, Groningen; ${ }^{3}$ Dept. of Hematology, Erasmus MC, University Medical Center Rotterdam, Rotterdam; ${ }^{4}$ Dept. of Rheumatology and Clinical Immunology, University Medical Center Groningen, Groningen, The Netherlands
}

10.1136/lupus-2020-eurolupus.54
Purpose This study aims to gain insight into the care provided to patients with antiphospholipid syndrome (APS) in The Netherlands and to identify areas for improvement from the perspective of both patients and clinicians.

Methods APS care was evaluated using qualitative and quantitative methods. Perspectives on APS care were identified using semi-structured interviews with medical specialists, patient focus groups and a cross-sectional, online patient survey. In order to examine differences in medical practice, medical records were reviewed retrospectively regarding clinical and laboratory manifestations, pharmacological treatment and management of disease in six hospitals throughout The Netherlands.

Results Fourteen interviewed medical specialists, fourteen focus group participants and 79 survey respondents participated in the study. 237 patients were included in medical record review. Only one-third of patients were diagnosed with APS within three months after entering specialist care. Diagnosis and management varied between centres and specialists. Almost $10 \%$ of triple positive patients did not receive any treatment at the time of medical record review. Major challenges according to specialists and patients were poor recognition of APS by health care professionals, fragmentation of care and lack of accessible, reliable patient education and psychosocial support.

Conclusion This study describes delayed diagnosis, variability in management strategies and a burden placed on patients to orchestrate their own care in APS care in The Netherlands. Of note, almost $10 \%$ of triple positive patients did not receive any treatment, despite this group of patients being at the highest risk of recurrent thrombosis.

Acknowledgements This work was supported by the Arthritis Research and Collaboration Hub (ARCH) Foundation.

\section{P6 RITUXIMAB EFFICACY IN CAPS}

Olena Yakymenko, Viktoria Vasylets, Viktor Klochko, Vladimir Savytskyi, Natalya Tikhonchuk. Rheumatological Dept., Multifield Medical Center of Odessa national medical university, Odesa, Ukraine

\subsection{6/lupus-2020-eurolupus.55}

Background Catastrophic antiphospholipid syndrome (CAPS) thrombotic microvasculopathy, characterized by involvement of systems and organs with formation of their insufficiency. Therapy of glucocorticoids and immunosuppressants with plasmapheresis and intravenous immunoglobulin demonstrates lack of effectivity. Currently, there are reports of rituximab effectiveness in APS and CAPS, but they are not numerous, especially CAPS.

Methods Assessment of the rituximab effectiveness in patients with resistant CAPS. We present the treatment results of two patients with CAPS, who were treated in our clinic.

Results Patient V., 21, was admitted in the early postpartum period. Diagnosis: Systemic lupus erythematosus, positivity for ANA: ds-DNA, Sm. Secondary antiphospholipid syndrome (positivity for $\beta 2$-glycoprotein-1, cardiolipin, lupus anticoagulant), catastrophic APS with multiorgan failure: epilepsy, sopor, psychosis; acute renal injury, signs of acute respiratory distress syndrome and hypocoagulation. SLEDAI score was 56 points, GAPSS - 17 points. Pulse therapy with methylprednisolone, cyclophosphamide, plasmapheresis and intravenous immunoglobulin wasn't effective enough. Rituximab course $375 \mathrm{mg} / \mathrm{m}^{2} 1$ time per week for 4 weeks was administered. Manifestations 